\title{
Denys CUCHE, La notion de culture dans les sciences
} sociales

\section{Anahy Gajardo}

\section{(2) OpenEdition}

1 Journals

Édition électronique

URL : https://journals.openedition.org/remi/5249

DOI : $10.4000 /$ remi.5249

ISSN : $1777-5418$

Éditeur

Université de Poitiers

\section{Édition imprimée}

Date de publication : 1 décembre 2010

Pagination : 218-221

ISBN : 978-2-911627-56-9

ISSN : 0765-0752

\section{Référence électronique}

Anahy Gajardo, «Denys CUCHE, La notion de culture dans les sciences sociales », Revue européenne des migrations internationales [En ligne], vol. 26 - $n^{\circ} 3$ | 2010, mis en ligne le 03 mai 2011, consulté le 14 avril 2022. URL : http://journals.openedition.org/remi/5249 ; DOI : https://doi.org/10.4000/remi.5249 


\section{NOTES DE LECTURE}

LARUELLE Marlène (Dir.)

Dynamiques migratoires et changements sociaux en Asie centrale. - Paris, Petra Éditions, 2010. - 320 p. ISBN : 978-2-847-43028-8

\section{Tarlène Laruelle est avec Virginie} MSymaniec directrice aux éditions parisiennes Petra d'une collection intitulée "Sociétés et cultures postsoviétiques en mouvement " et c'est précisément dans cette collection qu'elle fait paraître un ouvrage collectif avec pour titre: Dynamiques migratoires et changements sociétaux en Asie centrale. Cet ouvrage de 320 pages n'est pas le premier de la collection écrit ou dirigé par Marlène Laruelle puisque deux titres personnels déjà y figurent : La Quête d'une identité impériale. Le néo-eurasisme dans la Russie contemporaine, paru en 2007, et avec Catherine Servant : D'une édification l'autre. Socialisme et nation dans l'espace postcommuniste, paru en 2008. Cette jeune politologue est docteure de l'Institut National des Langues et Civilisations Orientales. Elle est spécialiste du néo-eurasisme russe, sujet de son doctorat paru sous trois titres, celui de l'édition Petra cité ci-dessus, l'un paru à L'Harmattan et l'autre aux Éditions du CNRS. Elle a également signé avec Sébastien Peyrouse deux ouvrages, l'un sur les Russes du Kazakhstan chez Maisonneuve \& Larose en 2004 et l'autre chez Autrement

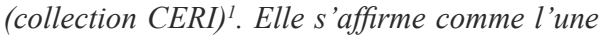
des figures montantes de l'étude des idéologies nationalistes du monde russe et ex-soviétique en philosophie politique et politologie.

1 L'Idéologie russe ou Comment penser l'empire ?, Paris, L'Harmattan, 1999 ; Mythe aryen et rêve impérial dans la Russie du $\mathrm{XXe}^{\circ}$ siècle, Paris, CNRS Éditions, 2005 ; La quête d'une identité impériale. Le néo-eurasisme dans la Russie contemporaine, Paris, Petra Éditions, 2007 ; avec PEYROUSE Sébastien, Les Russes du Kazakhstan. Identités nationales et nouveaux états dans l'espace postsoviétique, Paris, Maisonneuve \& Larose, 2004 ; Asie centrale, la dérive autoritaire. Cinq républiques entre héritage soviétique, dictature et islam, Paris, Autrement-CERI, 2006.
À ce titre, elle n'est donc pas au sens strict spécialiste des études sur le fait migratoire, mais de fait plusieurs de ses précédentes études, personnelles ou en partenariat, abordent les domaines complexes des migrations internes et internationales sur les territoires des anciennes républiques soviétiques, avec une prédilection pour l'Asie centrale au sens large, incluant le Kazakhstan. Sans être vierge, ce champ géographique de recherche reste encore mal connu du public, même relativement informé sur les tendances actuelles et récentes des flux migratoires sur l'immense territoire que représentent les quinze anciennes républiques. Deux raisons à cela, d'une part la réelle immensité des espaces considérés, rappelons que la Russie, même " esseulée ", couvre plus de 17 millions de kilomètres carrés, et le Kazakhstan, avec ses 15 millions d'habitants, plus de 2,7 millions de kilomètres carrés (soit l'équivalent à lui seul de l'UE!), immensité qui recèle des situations incroyablement diversifiées et mal connues, d'autre part, la relative nouveauté des questions liées aux mobilités et migrations dans des pays où la recherche en sciences humaines et sociales, sans être totalement absente de ces problématiques, restait dépendante de l'État central. Enfin, pourrait-on ajouter, la dislocation de l'URSS, accompagnée de quelques crises graves, mais moins nombreuses que ce que l'on aurait pu imaginer, a déclenché plusieurs mouvements massifs de mobilités forcées : Tchétchénie et Nord-Caucase, Karabagh, autour de la vallée de Fergana, conflits interethniques en Géorgie, etc. L'accident de Tchernobyl lui-même a poussé à l'exode des dizaines de milliers d'habitants et les Russes, passablement inquiets quant à l'avenir dans des républiques nouvellement indépendantes où ils n'étaient pas toujours bien vus par les autochtones " titulaires", ont souvent pris les devants en partant par " principe de précaution". Parmi les tout premiers ouvrages traitant de ces questions, on rappellera celui de Hisao Komatsu, Chika Obiya et John Schoeberlein qui 
était sans doute l'un des premiers disponibles ${ }^{2}$. Depuis les publications se sont multipliées, souvent sous les plumes d'Anatoli Vichnevsky, Jana Zayontchovkaya, Irina Ivaniukh, Vladimir Iontsev, Vladimir Mukomel, Vladimir Kolossov, etc. (Russie), Elena Sadovskaya (Kazakhstan), Saodat Olimova (Tadjikistan), etc. Les études commandées par les gouvernements républicains sur les "diasporas » se multiplient également, mais parfois dans une optique très dirigée. En d'autres termes, des "écoles" commencent à émerger en divers lieux de Russie ou dans les nouvelles capitales (Kiev, Astana-Almaty, Bishkek, Bakou). Mais il reste une grande marge de manouvre pour approcher l'état des connaissances occidentales (américaines, européennes, australiennes, japonaises, turques, inter alia) en ce domaine. La sociologie, l'anthropologie, plus que la politologie ou l'histoire restent, non pas à inventer, mais au moins à conforter tout en n'appliquant pas de façon simpliste et/ou néocoloniale des normes, méthodes et épistémologies venues d'ailleurs. L'étude des migrations a donc un avenir certain dans cette région du monde, reposant sur des faits évoluant extrêmement rapidement.

Au-delà d'une première introduction (pp. 9-20) rédigée par la coordinatrice, cet ouvrage aborde trois aspects plus spécifiques en trois parties : I. De la multiplicité des flux en Asie centrale, avec des textes d'Olivier Ferrando, Marlène Laruelle, Sophie Massot, Sébastien Peyrouse ; II. Évolutions sociales, transformations identitaires stratégies administratives, avec les contributions d'Asel Dolotkeldieva, Adeline Braux, Sophie Hohmann et Sophie Massot; enfin une partie III. L'impact des migrations sur les rapports de genre avec des articles de Madeleine Reeves, Luisa Piart, Nafisa Khusenova, Stéphanie Belouin. La première partie revient utilement sur l'historique assez méconnu en dehors des spécialistes de la Russie et de l'URSS des migrations russes et soviétiques sur le long

2 KOMATSU Hisao, OBIYA Chika et SCHOEBERLEIN John $(E d s)$ (2000), Migration in Central Asia: Its History and Current Problems, Osaka, The Japan Center for Area Studies, National Museum of Ethnology, 9, 255 p. terme et fixe les grandes lignes de mobilités en grande partie dictées par le politique plus que par l'économique, bien que, ici comme ailleurs, il soit parfois difficile de fixer des limites franches. Elle permet, avec Sébastien Peyrouse (Les anciens colons sur le départ. Les migrations des populations russophones, pp. 21-48) et Olivier Ferrando (Politiques diasporiques et flux migratoires : les Etats-nations et leurs co-ethniques de l'étranger, pp. 49-76) de mieux resituer les actuels courants migratoires dans un contexte plus large qui couvre les trois ères tsariste, soviétique et postsoviétique. De fait, migrations et mobilités ne sont pas nouvelles dans cet espace, mais il est resté l'image tenace d'une absence totale de liberté de déplacement hors migrations gérées par le politique, ce que viennent largement nuancer Sébastien Peyrouse et Olivier Ferrando par leurs mises au point. Au passage, remarquons l'usage d'une notion en rapide devenir, celle de "co-ethnique ", pour qualifier des populations de même langue et culture vivant dans des pays différents suite au découpage des frontières internationales. Ainsi pour ne citer qu'un exemple, les Ouzbeks présents en Kirghizie (sujet d'actualité !), Kazakhstan, Turkménistan, Russie, tous anciens citoyens soviétiques, et en Afghanistan, Chine, Turquie, Arabie saoudite et autres pays qui s'apparenteraient eux plutôt à des populations diasporiques au vu des circonstances historiques et politiques de leurs mobilités. La richesse du thème apparaît avec les textes de Marlène Laruelle (Le Kazakhstan, nouveau carrefour migratoire d'Asie migratoire, pp. 77-98) et Sophie Massot (L'exode rural comme migration de l'identité. Du kichlak ouzbek à Samakand la tadjike, pp. 99-122) montrant comment les situations se diversifient rapidement, ainsi du Kazakhstan pays d'émigration massive se retransformant en pays d'immigration, ou l'ambiguïté du cas ouzbek où les Tadjiks autochtones (descendants des sédentaires iranophones des oasis) se transforment en étranger de l'intérieur, mais où les ruraux ouzbeks finalement se " tadjikisent » en s'urbanisant, donc en porteà-faux par rapport à une politique nationale d'ouzbekisation (o'zbekchilik) qui en rappelle 
bien d'autres ${ }^{3}$.

Dans la seconde partie relative à des terrains plus localisés, reposant sur des recherches de thèses de doctorats en cours ou récemment soutenus, Asel Dolotkeldieva (Les migrants kirghizes à Moscou, politiques publiques, stratégies migratoires et réseaux associatifs, pp. 123-145, thèse en cours) nous propose une lecture d'une migration de travail peu qualifiée qui s'apparente à ce que l'Europe a connu avec les Maghrébins en France ou les IndoPakistanais en Grande-Bretagne : les droits les plus basiques des travailleurs aujourd'hui étrangers (alors qu'ils étaient des compatriotes auparavant, il n'y a pas si longtemps) ne sont guère respectés, corruption et xénophobie ont augmenté dans des proportions importantes sans que les autorités, tant des pays d'accueil et de résidence que des pays de départ, ne soient réellement en mesure de contrôler des situations encore largement inédites sur cet espace postsoviétique. Les migrants, ici précisément kirghizes, tentent de s'organiser pour à la fois se défendre et faire valoir leurs droits sur les deux bornes du champ migratoire. Adeline Braux (Les Azerbaïdjanais de Russie : une diaspora imaginée, pp. 146-168, à partir d'une recherche en cours) décrit une population presque otage des autorités de Bakou, mais face à un dispositif artificiel qui semble ne pas être efficace sur la population concernée, très passive face aux incitations du pays d'origine. L'auteur rejoint sur le cas spécifique de Moscou l'analyse un peu plus ancienne de Bayram Balci, tout aussi critique sur cette approche étatique du phénomène de diaspora4. Sophie Massot (Les migrations ouzbèkes vers Moscou, Séoul et New York: sacrifice ou rite de passage ?, pp. 169-188, thèse soutenue à Paris) nous livre un condensé d'une thèse ambitieuse et réussie sur trois terrains de recherche très éloignés mettant des migrants ouzbeks aux prises avec des réalités

3 On peut ici se référer au travail de PETRIC Boris-Mathieu (2002), Pouvoir, don et réseaux en Ouzbékistan postsoviétique, Paris, PUF-Le Monde (Partage du savoir), 298 p.

4 BALCI Bayram (2008), La place de la " diaspora 》 azerbaïdjanaise dans la politique de l'Azerbaïdjan postsoviétique : esquisse d'analyse, EurOrient, 28, pp. 185-204. économiques et sociales bien différentes. Alors que l'Ouzbékistan ne reconnaît pas la réalité du fait migratoire et l'importance de l'émigration, y compris dans son bilan économique et social, on voit ici les intéressés obligés de découvrir par leurs propres moyens des marchés très éloignés de leurs bases familiales. La question posée est celle de la migration, enjeu fondamental de la socialisation et de l'intégration sociale en Ouzbékistan. Sophie Hohmann (Migrations et enjeux socio-économiques et sanitaires de la guerre au Tadjikistan, pp. 189-216) revient sur une réalité plus dure encore, celle des suites à moyen et long terme d'un conflit violent, qui non seulement a fait de nombreuses victimes et de gros dégâts matériels, mais a aussi durablement déstabilisé une société qui était déjà l'une des plus pauvres et des plus défavorisées de l'URSS. Les aspects sanitaires (dégradation des soins et de la couverture sociale, épidémie $d u$ SIDA, etc.) sont le thème de spécialisation de l'auteur qui arpente un terrain des plus difficiles en collaboration avec les chercheurs tadjiks comme Madame Saodat Olimova.

Transition est faite par ce texte avec la partie suivante, car la population féminine $d u$ Tadjikistan est sans doute l'une des plus importantes victimes de la dislocation de l'URSS. Madeleine Reeves (Migrations, masculinité et transformations sociales dans la vallée de Sokh, pp. 217-246) et Nafisa Khusenova (La féminisation des migrations de travail tadjikes en Russie, pp. 278-296) nous décrivent des mutations importantes touchant, par le biais de l'émigration, même - et surtout! - lorsque l'épouse reste attachée au lieu d'origine. Soit elle est totalement victime de la situation nouvelle qui lui est imposée (avec le retour du patriarcat, de la polygamie), soit au contraire elle profite de nouvelles responsabilités pour transformer la société locale. Dans le cas des terrains étudiés par Luisa Piart (Des mondes marchands mobiles. Les commerçantes à la valise ouzbèkes, pp. 247-277, doctorat en cours) et Stéphanie Belouin (Projets et stratégies migratoires de femmes qualifiées à Tachkent, pp. 297-318), tous deux Ouzbeks, ce sont au contraire les femmes qui prennent l'initiative de l'émigration et viennent bousculer l'ordre 
établi des priorités et des hiérarchies. Mais dans ces deux cas, contrairement aux terrains tadjiks, les femmes bénéficient de formations et de statuts de départ bien plus enviables que les femmes du Tadjikistan rural.

Plusieurs questions transversales apparaissent ou sont posées par l'un ou l'autre article. La question des "co-ethniques " est dans l'ex-URSS une question centrale, car les frontières n'ont pas respecté - pouvaientelles d'ailleurs l'être réellement? - la répartition géographique des populations. Ceci est le thème de bien d'autres recherches en cours. Celle des "diasporas 》 en est directement issue et il faudrait réellement impulser des recherches sur ces politiques étatiques de construction de diaspora que pratiquent à des degrés divers Azerbaïdjan, Ouzbékistan ou Kazakhstan, à l'instar de la Russie. La question des migrations et droits de l'homme est cruciale, car corruption, xénophobie et nonrespects des droits parfois les plus élémentaires font dans ce vaste espace des ravages et malgré les efforts consentis par les gouvernements, au moins certains d'entre eux, les situations restent précaires, d'autant que la migration de travail a pris ici un tour inédit auquel les autorités ne sont guère habituées. Enfin, les conséquences de la migration sur les équilibres sociaux, en particulier sur les questions de genre, mériteront de vrais programmes de recherche pluridisciplinaires et sans doute internationaux.

Au total, un ouvrage frappant et passionnant par la diversité des situations étudiées, des terrains et des échelles d'analyse. Deux des originalités de cet ouvrage collectif sont la part donnée aux jeunes chercheuses en général (Adeline Braud, Sophie Hohmann, Sophie Massot, Stéphanie Belouin, Luisa Piart), et la présence de jeunes chercheuses ellesmêmes originaires de la région étudiée et très impliquée dans la relation entre la France et leurs pays respectifs (Asel Dolotkeldieva, Nafisa Khusenova). La directrice de l'ouvrage a su réunir une équipe jeune et diversifiée, l'ouvrage s'en ressent nettement et l'on comprend qu'une dynamique fondée sur une collaboration nouvelle est en train de s'affirmer sur un espace qui reste en grande partie inconnu hors des spécialistes de la région.

Stéphane de Tapia

Cultures \& Sociétés en Europe, Strasbourg Associé à MIGRINTER, Poitiers

\section{CUCHE Denys}

La notion de culture dans les sciences sociales [4e édition revue et augmentée]. — Paris : La Découverte, 2010. — 157 p. ISBN : 978-2-707-15883-3

Ceize ans après sa première publication en $\mathcal{N}$ 1994, la quatrième édition de l'ouvrage de Denys Cuche, La notion de culture dans les sciences sociales, paraît dans une version revue et augmentée (2010). Ce petit livre remarquable par son ambition synthétique, utile aux étudiant-e-s comme abondamment cité par les enseignants-chercheurs les plus confirmés, réussit le pari ô combien difficile de faire le portrait critique de l'usage de la notion de culture en sociologie et en anthropologie, en adoptant une perspective à la fois diachronique et synchronique. Rédigé dans un langage abordable, le panorama proposé expose avec complétude et rigueur les principaux enjeux et débats autour de ce mot polysémique et insaisissable, inhérent au vocabulaire des sciences sociales et humaines, en particulier dans l'étude des processus migratoires, mais pas seulement. Omniprésent dans le vocabulaire des sciences sociales, le terme a désormais aussi gagné le monde des médias, de la politique, de l'entreprise, des corporations professionnelles, du sport, de la jeunesse, de la formation, etc., autant d'univers qui se sont approprié le mot selon des logiques qui leur sont propres et qui appellent une analyse sociologique spécifique. Témoignant d'une certaine vulgarisation de l'anthropologie culturelle, l'expansion de cette notion ne date cependant pas d'aujourd'hui : il y a plus de vingt ans déjà, Marc Augé remarquait que le succès du terme de culture est à lui seul une donnée ethnologique (Augé, 1988, in Cuche, 2010 : 5). À ce titre, l'étude de l'usage de ce mot et de son appropriation par l'anthropologie et la sociologie éclairent de manière 
significative les logiques épistémologiques de ce que nous pourrions appeler - si nous ne craignions les abîmes sémantiques - nos "cultures disciplinaires ». Le triomphe du terme de culture soulève donc la question de l'intérêt d'instituer un courant de recherche plus systématisé à l'intérieur de nos disciplines autour de ce phénomène social, qui éclaire, par un double effet de miroir, les processus étudiés comme nos propres champs de recherche.

Composée de huit chapitres partiellement réorganisés, cette nouvelle édition de l'ouvrage de Denys Cuche gagne notamment en lisibilité et en clarté dans sa structure. Sur le plan formel, elle bénéficie d'une mise en page plus aérée et d'une table des matières qui inclut enfin les encadrés, au nombre de huit, qui rythment le livre et se focalisent sur une thématique ou un concept particulièrement significatifs dans l'histoire et la construction de la notion de culture en sciences sociales. Plus particulièrement, mentionnons à cet égard l'adjonction de deux nouveaux encadrés : l'un sur le concept de syncrétisme (pp. 72-73), dans lequel l'auteur retrace brièvement l'évolution du mot au cours du temps, et l'autre sur celui d'ethnicité (pp. 102-103). Longtemps cantonné au domaine de l'histoire des religions avant d'être utilisé pour caractériser différents phénomènes culturels, le concept de syncrétisme a conservé un sens négatif jusqu'au premier tiers du XXe siècle au moins. Les cultures dites "syncrétiques " désignaient alors des cultures suspectées d'impureté, contaminées par des éléments hétérogènes. Avec le courant de la postmodernité, le phénomène du syncrétisme est au contraire considéré de manière positive, célébrant le métissage et le mélange comme des buts à atteindre. Rejetant les notions de pureté ou d'authenticité culturelle, comme l'idée d'associer un jugement de valeur au phénomène du syncrétisme, l'anthropologie contemporaine, nous rappelle Denys Cuche, désigne par ce concept un phénomène universel, décrivant le processus de création d'un ensemble culturel original. Cependant, l'auteur souligne l'insuffisance du concept en lui-même pour rendre compte de la complexité des processus de métissage culturel et insiste sur l'importance d'identifier les formes de syncrétismes à l'œuvre - à travers par exemple la typologie réalisée par Bastide. Il invite aussi à procéder à une analyse fine de la situation socio-historique dans laquelle un type de syncrétisme apparaît. Dans le chapitre VI "Culture et identité », la mise en évidence du concept d'ethnicité par un encadré doit être relevée dans la mesure où sa quasi-invisibilité dans les éditions précédentes est significative de la réserve avec laquelle ce concept, " importé " de l'anthropologie anglo-saxonne, a été accueilli par l'anthropologie de langue française. Bien que l'approche relationnelle et situationnelle de l'identité et de l'ethnicité proposée par Barth (1969), abondamment cité par Denys Cuche dans ce chapitre, semble désormais avoir atteint un certain degré de consensus à l'intérieur de la discipline, il n'en demeure pas moins que l'anthropologie de langue française n'a intégré le mot " ethnicité » dans son vocabulaire d'usage courant qu'assez récemment. À cet effet, cet encadré sur le concept d'ethnicité, signé par Simon (1994), se focalise sur les ambiguités du concept en identifiant six points de flottement dans sa signification et comble une lacune à notre avis importante dans ce panorama de l'histoire et de la construction de la notion de culture dans nos disciplines. Plus globalement, la mention faite aux ouvrages de Poutignat et Streiff-Fenart (1995) et Martiniello (1995) dans la bibliographie de ce chapitre, absents dans les éditions précédentes, s'inscrit dans le même sens et contribue à une meilleure compréhension de l'articulation des concepts d'ethnicité et d'identité avec celui de culture.

Parmi les principaux ajouts à signaler dans cette édition de l'ouvrage de Denys Cuche, le plus perceptible est sans nul doute l'addition d'un nouveau chapitre sur la question des cultures d'entreprises et des cultures professionnelles (chapitre VII, pp. 115-125). Incluse à l'intérieur d'un autre chapitre dans l'édition précédente, cette partie devient ici un chapitre autonome, complété d'une partie inédite sur les grandes écoles françaises et la culture professionnelle militaire (pp. 121-125). L'auteur y retrace notamment le contexte d'émergence de la notion de "culture d'entreprise " aux 
États-Unis dans les années 1970, en France dès les années 1980, et analyse avec acuité l'appropriation de la notion anthropologique de culture par le langage du management comme une stratégie pour améliorer l'image de l'entreprise et favoriser l'identification et l'adhésion des salariés aux objectifs définis par les directions. C'est plus ou moins dans la même logique que celle du monde des entreprises que les grandes écoles françaises ont introduit dans leur vocabulaire la notion de culture afin de promouvoir à l'interne comme à l'externe une image positive de leur institution. Dans les deux cas, c'est souvent une acception réductrice, déterministe, culturaliste ou relativiste radicale de la culture qui est retenue. Un des mérites de ce chapitre est donc notamment de démontrer avec finesse, à l'aide d'exemples concrets tirés du terrain, l'instrumentalisation de la notion anthropologique de culture par les acteurs dans le but de servir leurs intérêts.

Signalons enfin l'ajout d'une partie sur la notion de "culture de pauvreté " (pp. 84-86), insérée dans le chapitre $V$, et celle d'une partie sur la question des rapports entre le développement et la culture (pp. 129-130) à l'intérieur du chapitre VIII consacré aux enjeux et aux usages sociaux de la notion de culture. Très succinctes, ces deux parties ont cependant le mérite de thématiser ces questions.

Centrée jusqu'ici sur les principales modifications apportées à l'ouvrage, cette recension serait cependant incomplète si nous ne rappelions, même très brièvement, le contenu global des différents chapitres qui composent ce livre. Le chapitre premier retrace la genèse sociale $d u$ mot en décryptant son évolution sémantique dans la langue française et décrit les rivalités francoallemandes autour des termes de "kultur 》 et de civilisation au XIXe siècle. Consacrés à l'invention et au triomphe du concept scientifique de culture, les chapitres deux et trois décrivent les principaux courants et auteurs ayant contribué à son approfondissement théorique (Tylor et la conception universaliste, Boas et la conception particulariste, Durkheim et les fondateurs de l'ethnologie française, Malinowsky et le courant fonctionnaliste, l'école "culture et personnalité », Lévi-Strauss et son analyse structuraliste, etc.). Centré sur le renouvellement profond du concept de culture lié à l'analyse des processus d'acculturation, le quatrième chapitre met particulièrement en évidence l'apport des travaux de Bastide. Les recherches sur les processus d'acculturation ont permis de dépasser une conception figée et homogène de la culture et conduit à une définition dynamique de cette notion ; à ce titre, ce chapitre nous paraît particulièrement essentiel. Dans le chapitre suivant, l'ouvrage analyse les hiérarchies sociales et culturelles au cour des rapports sociaux entre cultures et au sein des cultures et fait appel aux travaux de Marx, Weber, Lewis, Grignon et Passeron, de Certeau, Morin, Appadurai et Bourdieu, pour ne citer que les plus connus. Le rapport entre le concept de culture et celui d'identité est clarifié dans le chapitre VI, et les conceptions objectivistes, subjectivistes et relationnelles de l'identité culturelle y sont notamment analysées. Comme précisé plus haut, le chapitre VII est consacré aux cultures d'entreprises et aux cultures professionnelles et enfin, le dernier chapitre traite de quelques enjeux et usages sociaux de la notion de culture. L'auteur y aborde notamment la notion de "culture politique", la question du multiculturalisme et analyse de manière critique les notions de "cultures des immigrés » et «cultures d'origine ».

Plus que des détails de forme, la réorganisation partielle des chapitres et des parties qui composent cette nouvelle édition de l'ouvrage de Denys Cuche ainsi que les ajouts introduits reflètent l'extension de la notion anthropologique de culture à de nouveaux domaines de la vie sociale, ainsi que l'importance prise par certaines thématiques au sein des sciences sociales. En ce sens, la structure du livre et sa métamorphose progressive au fil des éditions nous renseignent, en soi, sur l'évolution constante de l'usage du terme de culture à l'intérieur de nos disciplines et champs de recherche. À ce titre, bien que l'auteur assume son choix (p. 8), l'absence d'une analyse de l'usage de la notion de culture dans le champ de la sociologie de l'art et de la création se fait remarquer. De même, il est surprenant que la 
sociologie de Claude Dubar sur les identités professionnelles et le processus d'acquisition de la culture qu'est la socialisation ne soit pas mentionnée dans cette édition accordant une place significative aux cultures professionnelles et aux sciences sociales de langue française. Enfin, un éclairage sur les usages de la notion d'" interculturalité ", un terme ayant gagné depuis les années 1970 le vocabulaire de la recherche en sciences sociales, celui du monde politique, de la formation et de la santé, aurait été, à notre avis, particulièrement pertinent. Mais il est vrai que l'ouvrage de Denys Cuche ne vise pas à l'exhaustivité et à juste titre; il serait sinon appelé à être constamment complété et renouvelé tant la notion de culture est, comme le processus qu'elle décrit, dynamique et en constante reconstruction. Sans aucun doute cependant, ce petit livre centré sur les principaux acquis de l'analyse culturelle propose en 157 pages un horizon complet, rigoureux et clair de la question abordée. L'ouvrage se lit et se relit aisément et peut sans conteste être considéré comme un manuel de référence pour les sciences sociales de langue française, un " classique » dans son genre.

Anahy Gajardo

Université de Genève, FPSE

FILHOL Emmanuel et HUBERT MarieChristine

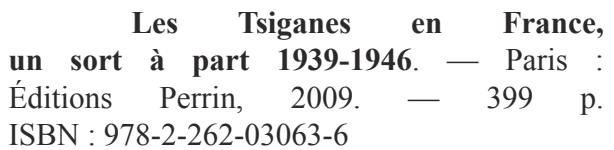

Dans un paysage où les recherches sur le sort subi par les Tsiganes pendant la Seconde Guerre mondiale sont encore émergentes en France, à la différence de pays comme l'Allemagne où l'historiographie du phénomène est plus ancienne et plus complète, ce livre foisonnant apporte une contribution importante.

L'internement a été le sort de 6000 à 6500 Tsiganes français, confinés dans trente camps d'internement pour " nomades", de 1940 à
1946. L'un des apports d'Emmanuel Filhol et de Marie-Christine Hubert est de montrer à quel point les persécutions des Tsiganes en France pendant cette période s'inscrivent dans une continuité historique débordant largement les dates de la Seconde Guerre mondiale. Le livre fait en particulier apparaître le poids des mesures législatives et administratives discriminatoires adoptées dans la première moitié du XXe siècle sur la mise en œuvre de l'internement. Ces dispositifs créent une catégorie de citoyens de seconde zone avec notamment la mise en place, en 1912, du carnet anthropométrique pour les nomades, instrument de surveillance qui servira ultérieurement aux autorités dans l'organisation de l'enfermement à partir de 1940. Ainsi, l'internement a été facilité par la suspicion d'office que les autorités publiques françaises entretenaient à l'égard des Tsiganes, suspicion qui leur a permis de passer très vite de l'omniprésence de l'idée de l'enfermement à sa mise en ouvre effective par les préfets, à qui revenaient l'organisation des interpellations et la gestion des camps. D'autres filiations historiques de la mise à l'écart des Tsiganes sont retracées : l'on découvre ainsi, par exemple, comment certains camps destinés aux réfugiés espagnols dans les années 1930 sont devenus des camps d'internement des Tsiganes quelques années plus tard, ce qui rejoint les constats des sciences sociales sur la permanence de la forme " camp » au XXe siècle.

Si la décision de l'internement appartint aux autorités militaires allemandes, son application fut entièrement assumée par l'administration française : d'ailleurs les préfets ne firent quasiment référence qu'à la loi française pour la mettre en ouvre. E. Filhol et M.-C. Hubert explorent à travers une minutieuse recherche des archives la manière dont l'internement a été orchestré, notamment à l'échelle régionale et locale. Le quotidien de ces camps, marqué par l'abandon, les privations extrêmes, et une surmortalité, notamment des enfants, est ainsi restitué dans ses détails. L'utilisation des archives donne des aperçus saisissants des destinées individuelles des internés et de leur diversité à travers des extraits de lettres des internés ou des membres de l'administration. 
Même si la mise en cuvre de l'enfermement fut parfois chaotique, sa logique sous-jacente, celle $d u$ regroupement, de la relégation avec parfois l'idée de rééducation autoritaire par le travail, a cuvré de manière puissante, condamnant les Tsiganes à vivre, avec des variations régionales et locales, une même réalité de l'internement. L'aspect familial de l'internement fut aussi d'importance, car cela revint à une prise en otage des familles et à une relégation d'autant plus complète que celles-ci ne purent espérer aucune aide extérieure.

Dans une Europe où les Tsiganes ont de manière générale subi les persécutions et l'extermination par les régimes nazis et leurs alliés, les spécificités du cas français sont dûment soulignées. Tout d'abord, l'internement des Tsiganes concerne, dans sa grande majorité, des citoyens français. Les lettres que les internés envoient aux préfets attestent de la présence des Tsiganes français dans les rangs de l'armée française pendant la Première Guerre mondiale, et des aspirations des internés à la citoyenneté et aux droits qui leur sont niés. Autre particularité française importante, l'internement de cette population ne vise pas explicitement l'extermination (les Tsiganes du Nord de la France, zone sous commandement militaire belge, seront, eux, déportés à Auschwitz et exterminés), mais bien la mise à l'écart. On retrouve aussi dans les documents émanant des autorités une volonté de sédentarisation ou de "rééducation par le travail » dont on se demande dans quelle mesure elle traduit les intentions ultimes des autorités. Enfin, dernière spécificité : les derniers Tsiganes durent attendre 1946 pour être libérés. Ils ne bénéficièrent d'aucune aide à leur sortie des camps et n'eurent de la part de l'État français aucune reconnaissance de leur sort après la libération. Dans les décennies suivantes, la loi ne récusa pas l'objectif de sédentarisation des Tsiganes. Le souvenir des camps fut "refoulé " par l'État, mais aussi dans l'aménagement urbain des communes, et, encore aujourd'hui, rares sont les marques commémoratives attestant des faits relatés dans ce livre.
À la suite de cet exposé édifiant, plusieurs questions demeurent, dont certaines sont évoquées en conclusion sans recevoir de réponse définitive. Comment comprendre le mélange de répression et d'abandon qui caractérise ce "sort à part 》 des Tsiganes en France, internés dans des conditions indignes, sans cependant qu'ils subissent une déportation massive? L'occupant allemand a délégué le sort des Tsiganes aux autorités françaises. Celles-ci, par " un enchevêtrement de circonstances ", notamment le fait que les moyens répressifs étaient monopolisés par la déportation des juifs, n'ont pas accordé de priorité à l'extermination des Tsiganes (pp. 350-352). Comment expliquer le caractère en apparence aléatoire de l'internement, puisque le livre nous apprend que les trois quarts des Tsiganes français et de nombreux Tsiganes étrangers $y$ échappèrent, et que certains internés pouvaient quitter les camps s'ils présentaient des garanties de "sédentarité »? La réponse semble être du côté de la spécificité de la catégorisation des Tsiganes par l'administration française, puisque la catégorie de "nomade" - et non la catégorisation raciale utilisée par les nazis en Allemagne pour définir les Tsiganes - a été retenue comme base pour l'identification et l'internement de cette population. Catégorie aux contours flous, car elle a aussi servi de base à l'internement de populations comme les forains ou les sans-abri, débordant ainsi le groupe initialement visé.

La manière dont la logique exterminatrice de la politique raciale allemande et celle d'exclusion et de refus du "nomadisme " de l'État français se sont imbriquées fait jaillir de nombreux questionnements, et le travail d'Emmanuel Filhol et de Marie-Christine Hubert ne manquera sans doute pas de stimuler les futures recherches.

Alexandra Nacu Centre de Sociologie des Organisations CNRS - Sciences-Po 
GUÉRIN-PACE France et FILIPPOVA Elena (Dir.)

Ces lieux qui nous habitent.
Identité des territoires, territoires des
identités. - Paris, La Tour d'Aigues,
Ined-éditions de l'Aube, 2008. - 276 p. ISBN : 978-2-752-60494-7

Doursuivant le dialogue initié en juin 2007 à l'INED entre géographes, démographes, sociologues, anthropologues et historiens, l'ouvrage revient sur la pertinence, en ce début de XXIe siècle, du lien entre les constructions identitaires individuelles ou collectives et leurs inscriptions spatiales. Dans un contexte mondialisé où l'individu multiplie le plus souvent ses appartenances et revisite les contraintes de l'espace-temps, il est en effet légitime de s'interroger sur la capacité d'affiliation et d'identification du territoire. Tentant de répondre à cette vaste question, les auteurs placent l'individu au cour de leur réflexion et décryptent des modalités par lesquelles certains lieux sont "investis " au gré des parcours de vie. Et chacune des quinze réflexions met en évidence la pluralité des configurations et des usages possibles du territoire. En réalité, par la diversité même des terrains abordés, des échelles géographiques et des méthodologies retenues, ces textes témoignent de la vigueur du territoire comme vecteur identitaire et de sa prégnance, tant dans des discours institutionnels qu'individuels. Ainsi posé, le débat sur "ce qui fonde le sentiment d'appartenance à un territoire ", pour reprendre les termes des directrices de l'ouvrage, dépasse avec bonheur le seul champ géographique.

Cependant, la diversité des études de cas présentées, qui constitue certainement une des qualités de l'ouvrage, aurait pu permettre de retravailler en profondeur les notions de territoire et de territorialité. La notion de lieu est, quant à elle peut mobilisée. Or, si le format " actes de colloque " de l'ouvrage justifie le choix transversal d'un angle descriptif, on peut toutefois regretter une discussion théorique plus étoffée sur ces notions, certes, mouvantes. Pour autant, cet ouvrage collectif n'est pas dépourvu de ligne générale : celle de l'auscultation des mécanismes de l'appropriation spatiale, d'un territoire considéré à travers le prisme des pratiques et des connaissances. Et tous les auteurs privilégient la piste de l'identité comme construction et résultat d'un processus relationnel plutôt que comme identité catégorielle nécessairement figée. Ils sont ainsi plusieurs à interroger les discours identitaires et les valeurs qu'ils mobilisent; quand d'autres examinent le territoire par les pratiques individuelles et collectives. Ces interrogations sur « la composante spatiale des identités " (première partie) et sur "l'invention des territoires identitaires" (deuxième partie) structurent l'ensemble de l'ouvrage et se résolvent dans la reconnaissance d'une relation complexe entre identités et territoires (troisième partie).

Ces intitulés de partie reflètent d'ailleurs l'intrication inévitable de la géographie et du social dans toute tentative de définition des lieux qui nous habitent. Ouvrant les débats les coordinatrices proposent une réflexion sur le processus de " mise en place des appartenances territoriales ». Leur approche est à la fois géographique et psychosociologique : elles s'interrogent sur l'essence des lieux d'attachement et cherchent à mettre en évidence la nature du lien qui attache un individu à des lieux. Leur analyse croise l'enquête nationale de l'INSEE réalisée en 2003 sur la construction des identités (l'enquête Histoire de vie) et leurs propres entretiens réalisés dans différentes régions françaises. Émaillant largement leur article par des extraits d'entretiens, elles veulent illustrer comment les contours $d u$ " chez-soi » varient d'une personne à l'autre et selon les circonstances. Cependant, et de manière peut-être un peu contradictoire, leur étude tend à montrer qu'en dépit des parcours, il n'existe qu'un nombre limité de "modèles d'appropriation spatiale».

Yannick Sencébé prolonge cette question de l'appartenance territoriale par un texte bref et bien écrit sur l'association couramment faite entre Modernité et "déclin de l'appartenance en tant que lien social collectif et durable ». En jouant, dans le titre de son article, sur déclindéclinaison, la sociologue suggère d'ailleurs 
que ce qui apparaît comme un affaiblissement des appartenances n'est peut-être en réalité que la multiplication des modalités de liens aux lieux et aux groupes. Appartenance et territoire sont donc les pierres angulaires d'une réflexion qui fait l'hypothèse de logiques d'appartenance variable d'une part à l'espace de l'inscription spatiale (l'espace des lieux) et d'autre part à celui des flux. L'auteur en dénombre quatre: selon que les lieux d'appartenance font liens, que les liens d'appartenance font lieux, que les liens et les lieux d'appartenance sont dissociés ou que, finalement, le présent définit les lieux et les liens d'appartenance. Disant cela, l'auteur souligne à quel point mobilité et ancrage (un thème cher à M. Hirschhorn et J.-M. Bertholot) fondent, aujourd'hui, des territoires en tension entre " activisme territorial actuel » et régime de mobilité.

Si c'est toujours le lien spatial que MarieLaetitia Helluy-des-Robert aborde dans son article, elle en traite quant à elle par ces différents "degrés». Ce qu'elle qualifie d'attachement spatial d'une personne à un espace en est le premier. Mais la relation au lieu peut être plus fortement chargée d'affect et l'attachement spatial fait place à de "l'appropriation spatiale ». Les lieux deviennent alors une source d'inspiration qui "colore " la vie des individus, par opposition, probablement, à un espace indéfinissable et indistinct. Au-delà, le lieu signifiant est incorporé et devient un territoire qui " code l'identité, statutaire ou intime, des individus qui s'en réclament». L'auteur repose toutefois la question de la force $d u$ terme même ; celle d'un mot qui n'appartient le plus souvent qu'à un vocabulaire scientifique qui veut rompre avec le sens commun. Cette question traverse également, bien que d'une autre manière, l'article de Catherine Armanet sur le lieu de sépulture, et en particulier la sépulture de cendres. La géographe propose en effet le lieu de sépulture comme un critère tangible et révélateur de l'identité territoriale. Comme marqueur ou énoncé de l'attachement au lieu, celui-ci est, il est vrai, à de nombreux égards, définitif. Nous interrogeant sur la pertinence de la sépulture à traduire l'identité territoriale, l'auteur propose de fait une réflexion sur ce qu'est l'identité territoriale. Si la sépulture, et en particulier la sépulture de cendres, représente un cas limite de la réflexion sur l'identité territoriale - il peut à la fois nier toute expression identitaire spatiale ou, au contraire, affirmer un attachement territorial précis (par la dispersion ou l'inhumation de cendres dans un lieu particulier) -, l'auteur voit dans l'augmentation croissante de choix de lieux particuliers pour déposer les cendres funéraires la diversification de l'expression de la pluralisation de l'identité territoriale.

De cet article à celui de Vincent Veschambre sur le patrimoine il n'y a étonnamment qu'un pas et c'est d'ailleurs un autre intérêt de cet ouvrage. Venant clore la partie sur "la composante spatiale des identités ", l'auteur discute la notion d'identité et explicite le choix de lui substituer des termes tels que "construction identitaire » et « identification ». Il « entre» par le patrimoine pour réfléchir aux processus de construction identitaire qui permet notamment d'articuler les deux dimensions fondamentales que constituent l'espace et le temps. Le patrimoine constitue en effet un support privilégié des constructions identitaires dans la durée et il constate que le recours à ce dernier est exacerbé "dans des sociétés où la mobilité, extrêmement valorisée dans une économie flexibilisée " concerne une part importante de la population. Il conclut par une mise en garde contre la réduction des processus d'identification aux seules «identités territoriales ».

C'est à Julien Aldhuy que revient d'ouvrir la seconde partie de l'ouvrage avec son article sur les liens qui se nouent entre l'identité du territoire landais et le département des Landes. L'auteur propose le couple "identité territorialité " plutôt que celui d'"identité territoire ». En effet, pour cet auteur comme pour de nombreux géographes, le territoire n'est qu'une des modalités de cette relation. L'article de Ronan Le Coadic, qui étudie le lien territoire identité en Bretagne montre comment de "la première modernité » (XIXe et première moitié $d u X X e)$ à nos jours l'identité individuelle ou collective est une construction évolutive inscrite dans un contexte socio-historique d'interac- 
tions multiples. Il part de la création de la carte d'identité, et avec elle du territoire national, pour discuter de "l'identité à la carte et des territoires superposés 》 de la période contemporaine. L'auteur conclut son article en notant que les individus disposent aujourd'hui d'un vaste éventail d'identifications au territoire et mêlent, voire superposent, les appartenances. Dépassant cependant l'idée de désordre contemporain, il tente la distinction des différents territoires d'identification selon qu'ils sont des territoires légitimes pour les régions administratives et d'aménagement du territoire imposés par les pouvoirs publics ; des territoires qui résistent pour les espaces qui véhiculent les rémanences de valeurs pré-modernes et notamment religieuses; des territoires de projet portés par des projets collectifs ou individuels.

L'exemple de la Costa Serena proposé par Philippe Pesteil se situe au croisement de ces différents ensembles. Dans le contexte corse de réaménagement territorial en micro-régions, il cherche à savoir quel peut être l'impact sur les populations de la superposition de découpages territoriaux "hérités, construits en ou construction ». Il se demande si les nouveaux espaces sont véritablement vécus et appropriés par les populations ou s'il ne s'agit que d'une grille technique d'aménagement du territoire. Sylvie Sagnes dans son article sur la tentative de rebaptiser la région Languedoc-Roussillon en "Septimanie », à l'arrivée d'un nouvel élu à la tête du conseil régional (2004) est assez proche. Elle retrace l'essor contemporain de cette notion - la Septimanie - et nous fait voir les intérêts divergents de ses promoteurs. Avec cette "tentative de greffe toponymique" ratée puisqu'un an seulement après sa proposition, elle doit être retirée, face aux différentes protestations, l'auteure souligne comment cet essai de mutation géonymique participe d'un phénomène général de "retour au local " souvent traduit par des "recyclages et créations onomastiques ».

Dans l'article d'Éric Auburtin, il est également question de construction régionale puisqu'il traite de la construction d'une identité transfrontalière dans la Grande Région
SarLorLux, sarroise, lorraine et luxembourgeoise. Il nous conduit au cour de l'action administrative et politique dans la construction territoriale. Cependant, si la construction de l'Europe passe par la production de " nouveaux objets territoriaux transfrontaliers ", l'auteur rappelle que la réalité transfrontalière est également faite de la mobilisation d'acteurs économiques et associatifs. Considérant l'espace transfrontalier comme un espace vécu, il cherche à comprendre le rôle de la frontière dans la territorialité d'individus "à la fois résidents et passeurs d'une frontière ». Observant l'usage que font les travailleurs lorrains au Luxembourg, et la ressource, avant tout professionnelle, qu'elle peut constituer, il propose d'envisager la frontière comme un " ourlet » qui s'étend en fonction de l'influence des métropoles frontalières sur leurs espaces périphériques.

C'est sur l'articulation des identités et des territoires que se conclut l'ouvrage. L'article d'Hervé Le Bras traite de la question de l'ethnicité et de l'intolérance dans les relations de voisinage. Il reprend le modèle de l'économiste Thomas Schelling selon qui de faibles intolérances entre groupes au sein d'une même agglomération suffisent à produire de la ségrégation spatiale. L'auteur prolonge ce modèle en donnant à la fois raison et tort à Schilling en montrant que "de faibles intolérances produisent presque toujours des structures fortes", mais que ces structures ne peuvent être interprétées de manière univoque. Yves Guermond dans son article "L'identité nationale en question" souligne à son tour que la délimitation conduit à l'édification de frontières qui ensuite institutionnalisent les identités. Il propose une réflexion sur une identité nationale " en contrepoint des "géographies des frontières" " qui ne soit pas fondée sur "la fatalité d'un découpage fixe des "États-nations" ". Ces deux articles se font donc écho en rappelant que la frontière et sa naturalisation participent largement de l'institution du groupe.

Kamel Kateb revient sur l'instauration, en 1882, de l'état civil colonial par la France en Algérie. Il explique comment l'obligation 
d'enregistrement à l'état civil selon le système français d'identification des personnes et leur ancrage subséquent au territoire a aussi été une vaste action politique de "désancrage" de populations tribales de leur territoire. Par la sédentarisation des populations nomades, $c$ 'est en réalité la rupture du lien entre population et territoire ancestral que le gouvernement français semble vouloir opérer. En effet, pour sortir la propriété " du cadre collectif dans lequel elle était enserrée, il était nécessaire de dégager la population de l'organisation sociale constituée par la tribu ». Mais en transformant le référentiel identitaire des indigènes algériens, l'administration française a également ouvert la voie d'une "identité plus globale de type national ».

Dans ce système à dominante sédentaire qui est le nôtre, et malgré les déclarations de fin du territoire et de la territorialité de certains théoriciens de la globalisation, on ne peut ignorer que le signe de reconnaissance d'identités "propres " continue d'être en relation avec la possession d'un territoire. Christian Giordano rappelle à cet égard que le "nouveau » régionalisme européen n'a pas évacué la dimension identitaire du territoire. Pourtant, dans les sociétés postcoloniales, et dans des constellations historico-sociales bien définies, le facteur territorial peut jouer un rôle moins important dans la fabrication des identités. Sur l'île de Pulau Penang en Malaisie, dans la ville de George Town par exemple, chaque groupe possède "ses institutions et ses structures à l'intérieur d'un territoire bien défini ». La subdivision voulue par les Britanniques en quartiers et en espaces sociaux ethniques a en effet favorisé l'émergence d'identités fortement territorialisées qui s'expriment pourtant aujourd'hui par des représentations et des discours publics de "l'unité dans la diversité ", tant au niveau national que local. Le cas de Penang et de la Malaisie montrerait que, au contraire des contextes européen et nord-américain où la territorialisation de l'appartenance est souvent considérée comme la condition d'une cohabitation multiculturelle pacifique, la déterritorialisation des affiliations ethnoculturelles sans l'effacement des frontières communautaires a favorisé la cohésion sociale en créant des rapports interethniques multiples.

Le dernier article de l'ouvrage porte sur le quartier du Marais à Paris et sa représentation comme espace communautaire gay et clos. Marianne Blidon aborde ce qu'elle analyse comme un décalage entre discours (anticommunautariste) et perceptions (variées) de cet espace et de sa dimension identitaire. Elle propose une géographie du Marais à la fois plus sélective et mieux intégrée dans la ville et interroge la pertinence du couple "identité " et " espace" : aborder le Marais comme un quartier gay, c'est, nous dit-elle, ne pas voir la pluralité de cet espace. L'auteure s'attache à montrer que l'attrait du quartier se joue surtout dans la juxtaposition de communautés, au cœur d'un espace historique à l'architecture exceptionnelle.

Pour conclure la présentation de cet ouvrage à recommander aux lecteurs, il nous semble que, l'effort de clarification et de catégorisation sur les liens entre identification et territorialité, qui a retenu les auteurs, aurait gagné en force en intégrant une synthèse de l'ensemble des développements proposés. Le questionnement qui traverse ce livre sur la place des lieux dans nos constructions identitaires et territoriales, individuelles et collectives n'en aurait certes pas été épuisé pour autant, mais il en aurait peut-être été plus nourri, dans un contexte mondialisé où les lieux continuent, à n'en pas douter, de nous habiter.

Caroline Rozenholc

Migrinter, Université de Poitiers

MAZZELLA Sylvie (Dir.)

La mondialisation étudiante.

Le Maghreb entre Nord et Sud.

- Paris, Karthala, 2009. - 404 p.

ISBN : 978-2-8111-0307-1

L'ouvrage dirigé par Sylvie Mazzella de recherche intitulé Étudiants étrangers au Maghreb et dans l'espace euro-méditerra- 
néen. Vers quelle internationalisation de l'enseignement supérieur et vers quelle circulation des compétences ? Dirigé par l'Institut de Recherche sur le Maghreb contemporain, il a réuni une vingtaine de chercheurs et de doctorants marocains, algériens, tunisiens et français. Les vingt-et-une contributions qui le constituent offrent une documentation inédite sur les étudiants maghrébins à l'étranger et les étudiants étrangers au Maghreb.

Les deux points forts de l'ouvrage résident d'une part dans l'effort de comparaison qui permet de livrer un état des lieux situé des transformations de l'enseignement supérieur au Maghreb et d'autre part dans les démarches méthodologiques qui combinent approche statistique, institutionnelle et socio-anthropologique.

Comment les politiques d'accueil des étudiants maghrébins en France ont-elles évolué ? Les premiers chapitres traitent de l'évolution du cadre institutionnel et des dispositifs qui encadrent l'immigration étudiante. Valérie Erlich (Étudiants « expatriés 》 versus étudiants " résidents ». Profils et logiques migratoires des étudiants maghrébins en France, pp. 55-77) identifie deux logiques migratoires: la première est une "logique d'expatriation " chez des étudiants nés et ayant étudié à l'étranger, qui cherchent à valoriser leur qualification, l'autre est une "logique d'immigration " chez des étudiants étrangers résidant en France et qui souhaitent s'y installer. Cette recherche est complémentaire de celle de Constance de Gourcy (Partir pour revenir ou partir pour quitter? Le projet d'études des étudiants algériens entre autonomie et attaches, pp. 97-115) qui montre que le projet d'études ne peut se comprendre uniquement comme un choix stratégique personnel : démarche coûteuse et longue, l'élaboration d'un projet migratoire doit se replacer dans une logique familiale.

Alexis Spire aborde de façon détaillée la sélection des étudiants candidats à l'émigration vers la France (Contrôler et choisir. La sélection des étudiants candidats à l'émigration vers la
France, $p p$. 79-95) dans l'enquête qu'il a menée, en observant une cinquantaine d'auditions de candidats au départ et en menant une série d'entretiens avec les acteurs du consulat et des services culturels de l'ambassade. Depuis 2005 une nouvelle procédure issue de la politique de "l'immigration choisie » a été mise en place : le dispositif intitulé "Campus-France " instaure une sélection des dossiers avec notamment pour critères, le " sérieux » et "la réalité du projet des étudiants "; le dossier des candidats est aussi soumis à une "évaluation pédagogique et linguistique ». Ces procédures ne sont pas sans présenter de nombreux points communs avec les autres recherches antérieures réalisées par l'auteur ${ }^{5}$ : opacité des informations détenues par les services consulaires, opacité de la prise de décision, division du travail entre services et déresponsabilisation de chaque agent dans la prise de décision.

Quels sont les enjeux pour les universités $d u$ Maghreb du processus de Bologne? À quelles conséquences en termes d'harmonisation pédagogique les pays doivent-ils faire face? C'est à ces deux questions que la seconde partie cherche à répondre. La démarche est d'abord descriptive et la comparaison des réformes de l'enseignement supérieur permet de mieux comprendre les enjeux de l'internationalisation liés à la mobilité des étudiants.

Youcef Berkane (L'internationalisation de l'enseignement supérieur : quelles perspectives pour l'université algérienne ?, $p p$. 149-160) apporte un éclairage sur l'évolution de l'enseignement supérieur en Algérie. Le système LMD en place dans les pays de l'Union européenne a été adopté de façon rapide dans l'enseignement supérieur algérien à la rentrée universitaire 2004-2005 dans dix établissements. En 2008 il concerne quarante-et-un établissements. Mais comme le montre Zineddine Berrouche (Mise en place du LMD en Algérie : entre nécessité et résistances, pp. 161-172), cette évolution ne doit pas masquer les nombreuses résistances

5 SPIRE Alexis (2008) Accueillir ou reconduire. Enquête sur les guichets de l'immigration, Paris, Raisons d'agir, 2008, $124 \mathrm{p}$. 
dans son application du côté des enseignants, des étudiants et de l'administration. Florian Kohstall (Une internationalisation concurrentielle. Les réformes de l'enseignement supérieur en Égypte et au Maroc, $p p$. 173-186) montre quant à lui qu'en Égypte et au Maroc, les réformes de l'enseignement supérieur mises en cuvre de "façon partielle et sélective » (p. 184) peinent à porter leurs fruits. Fethi Rekik (LMD, employabilité et nouvelles mobilités des étudiants tunisiens, $p p$. 199-217) pointe dans son article, sur la situation en Tunisie, les contradictions des réformes LMD : censées épauler la mobilité des étudiants, selon l'esprit de Bologne, et développer leur employabilité, leur application génère au contraire une sélectivité et finalement une restriction de la mobilité. De plus, alors que le discours officiel prônait une application mesurée et progressive, il constate que la réforme a été adoptée à la hâte.

La troisième partie de l'ouvrage est consacrée aux étudiants étrangers au Maghreb. Les différentes contributions apportent des informations précieuses et détaillées sur les projets migratoires de ces étudiants, leur expérience universitaire et leurs modes de vie. Parmi eux on trouve majoritairement des étudiants venus d'Afrique subsaharienne et du Moyen et Proche-Orient. Les deux premiers chapitres fournissent des données statistiques sur ces populations estudiantines. Au Maroc, si la présence des étudiants étrangers est limitée (ils représentent 2 à $3 \%$ des étudiants nationaux) depuis le milieu des années 1980 cependant, on observe que les effectifs d'étudiants étrangers dans l'enseignement supérieur sont en constante hausse dans les universités publiques : en 2005-2006, leur nombre s'élèverait à 9 236. Ce sont les étudiants originaires de l'Afrique subsaharienne qui sont les plus nombreux $(60,3 \%)$, contre $11,6 \%$ en provenance du Moyen-Orient et $17 \%$ d'autres pays du Maghreb. En Tunisie, l'effectif des étudiants étrangers n'a cessé d'augmenter depuis la fin des années 1970 ; en trente ans, leur nombre a été multiplié par huit. Comme au Maroc, les étudiants originaires d'Afrique subsaharienne sont les plus nombreux, lorsque l'on réunit les deux secteurs d'enseignement supérieur.
L'objectif de cette troisième partie est bien de montrer que les pays du Maghreb sont des producteurs d'étudiants diplômés. Les différents auteurs des monographies présentées ici dressent un portrait composite de la réalité. Sophie Bava décrit la situation des étudiants africains à Alger et au Caire, Sylvie Bredeloup celle des étudiants burkinabè et Mohammed Abderahmane Ould Ahmedou Yacoub (Les étudiants mauritaniens en Tunisie. Formation universitaire et stratégies familiales, pp. 283-298) celle des étudiants mauritaniens. A partir d'une enquête réalisée en 2007 et 2008 sur soixante-neuf étudiants à Sousse et Monastir, l'auteur s'intéresse aux enfants de familles aisées dont la majorité sont des filles et fils de personnalités politiques et de décideurs économiques. Leur migration pour études doit être comprise comme le résultat d'un projet collectif dans lequel les parents jouent un rôle majeur. Hassan Boubakri et Makrem Mandhouj (Les étudiants marocains en Tunisie. Choix des filières médicales et stratégies de reproduction sociale, pp. 299-316) analysent eux le choix stratégique de contournement que font des étudiants marocains en Tunisie en choisissant des filières médicales sélectives, filières auxquelles ils ne peuvent accéder dans leur pays.

Au total, on ne peut que conseiller vivement la lecture de cet ouvrage collectif, qui offre un point de vue complet, circonstancié et minutieux sur les formes d'internationalisation de l'enseignement supérieur au Maghreb. La reconstruction des contextes spécifiques aux projets migratoires, l'attention à leurs dimensions familiales, la multifocalité des analyses empiriques permettent aux auteurs de donner des résultats d'enquêtes nuancés et précis. On soulignera pour conclure que l'introduction de Sylvie Mazzella et les introductions de chaque partie viennent judicieusement synthétiser les apports des différentes contributions et organiser la lecture.

Frédérique Giraud GRS, UMR 5040 École Normale Supérieure de Lyon 